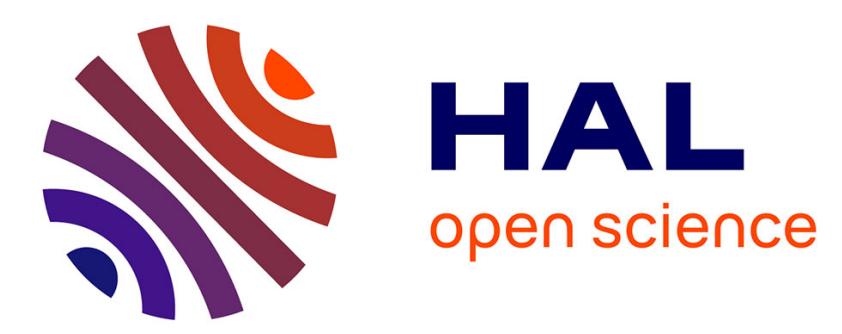

\title{
Essais d'hybridation interspécifique entre Lupinus albus L. et Lupinus mutabilis Sweet. Influence des conditions de culture et du génotype
}

\author{
Elisabeth Vuillaume, Tine Hoff
}

\section{- To cite this version:}

Elisabeth Vuillaume, Tine Hoff. Essais d'hybridation interspécifique entre Lupinus albus L. et Lupinus mutabilis Sweet. Influence des conditions de culture et du génotype. Agronomie, 1986, 6 (10), pp.919924. hal-00884840

\author{
HAL Id: hal-00884840 \\ https://hal.science/hal-00884840
}

Submitted on 1 Jan 1986

HAL is a multi-disciplinary open access archive for the deposit and dissemination of scientific research documents, whether they are published or not. The documents may come from teaching and research institutions in France or abroad, or from public or private research centers.
L'archive ouverte pluridisciplinaire $\mathbf{H A L}$, est destinée au dépôt et à la diffusion de documents scientifiques de niveau recherche, publiés ou non, émanant des établissements d'enseignement et de recherche français ou étrangers, des laboratoires publics ou privés. 


\title{
Essais d'hybridation interspécifique entre Lupi- nus albus L. et Lupinus mutabilis Sweet. Influ- ence des conditions de culture et du génotype
}

\author{
Elisabeth VUILLAUME \& Tine HOFF
}

I.N.R.A., Station d'Amélioration des plantes, Centre de Recherches de Dijon, BV 1540, F 21034 Dijon Cedex

\begin{abstract}
Nous avons réalisé des hybridations interspécifiques entre Lupinus albus L. ( $९$ ) et Lupinus mutabilis Sweet ( $\odot$ ), en serre et au champ, avec divers génotypes dans chacune des deux espèces.

Il n'y a pas d'effet, sur les taux de nouaison, des conditions de culture des plantes. Par contre, en serre, nous observons toujours la nécrose des ovules (fécondés ?), 3 à 4 semaines après la réalisation du croisement, alors qu'au champ, un certain nombre d'ovules (fécondés?) se sont développés jusqu'à donner des graines de taille presque normale, mais plates.

Les coupes histologiques réalisées ne nous ont pas permis de voir si ces graines contenaient un embryon ayan avorté plus ou moins précocément.

Par ailleurs nous observons des effets génétiques sur les taux de nouaison et sur les rendements en graines susceptibles de contenir des embryons plus ou moins développés.
\end{abstract}

Mots clés additionnels : effets génétiques. Lupinus albus $L$. and Lupinus mutabilis Sweet.

Interspecific crosses were made between Lupinus albus $\mathrm{L}$. as the seed parent and Lupinus mutabilis Sweet as the pollen parent, in the greenhouse and in the field, with different genotypes of the two species. Fruit set was the same in the greenhorise and in the field but, in the greenhouse, ovules always died 3 to 4 weeks after the cross was made. In the field, some ovules developed to give seeds of almost the same size as selfed seeds. As these were flat, they appeared to be empty and we were unable, by histological studies, to observe whether they contained an aborted embryo or not. We also observed genotypic effects on fruit set and on the yield of seeds of quite normal size.

Additional key words : genetical effects.

\section{INTRODUCTION}

L'adaptation aux exigences culturales de notre pays de l'espèce Lupinus mutabilis Sweet, originaire des hauts plateaux des Andes, pourrait être un des moyens de réduire notre déficit en protéines pour l'alimentation animale. Cette espèce possède en effet des grains ayant une teneur en protéines de $48 \%$ à $52 \%$ et une teneur en matières grasses de $11 \%$ à $16 \%$ (LENOBle \& PAPINEAU, 1977 ; GladSTONE, 1978 ; WiLliaMS, 1984). La sélection de cette espèce consiste actuellement à trouver des génotypes qui présentent les caractères suivants :

- précocité de floraison et de maturation des gousses associées à une floraison non remontante ; ble ;

- potentialité de rendement la plus élevée possi-

- absence d'alcaloïde ;

- résistance à l'anthracnose provoquée par Colletotrichum gloeosporioïdes.

En Amérique latine, plusieurs équipes sélectionnent 
L. mutabilis, parmi lesquelles l'équipe de VON BAER au Chili et l'équipe de BLANCO au Pérou (VON BAER, 1984 ; BLANCO, 1984). Les géniteurs retenus par ces équipes sont, quelquefois, très intéressants pour les sélectionneurs européens ; c'est ainsi que VON BAER et GROSS (1983) ont obtenu des géniteurs à faible teneur en alcaloïde.

En France la variabilité naturelle de cette espèce, pour les caractères cités, est étudiée, depuis une dizaine d'années, par LENOBLE à Lusignan, BERTHAUT d'abord puis VUILLAUME à Dijon. Jusqu'à présent nous n'avons pas trouvé de génotype adapté à l'une quelconque de ces exigences. En GrandeBretagne, WILliAMS a réussi à sélectionner, dans des populations récoltées au Pérou, des génotypes à floraison précoce mais qui reste remontante.

Nous avons aussi très largement utilisé les moyens du laboratoire de mutagenèse de Dijon et, par traitement au M.S.E., nous avons réussi à diminuer la teneur en alcaloïde, la ramenant d'environ $3 \%$ à $0,5 \%$ et même $0,2 \%$. Cependant une partie du matériel suivi à Dijon pour sa faible teneur en alcaloïde est issu de croisement entre des génotypes sélectionnés par LEN@BLE et des génotypes à faible teneur en alcaloïde sélectionnés par VON BAER au Pérou. Un génotype à faible teneur en alcaloïde a été obtenu par WILliams et al. (1984), après traitement mutagène. D'après les premiers résultats, la faible teneur en alcaloïde serait sous le contrôle d'un gène récessif.

L'exploitation de la variabilité «naturelle ou induite » de cette espèce apparaît donc insuffisante pour pouvoir résoudre les problèmes rencontrés dans sa domestication.

L'hybridation interspécifique du lupin andin avec les lupins européens pourrait être le moyen de résoudre de tels problèmes de variabilité.

Il existe un certain nombre d'espèces de lupin cultivées en Europe depuis des générations comme plantes fourragères. Parmi ces espèces, les sélectionneurs français ont choisi de travailler sur Lupinus albus $\mathrm{L}$. non plus comme fourrage mais comme légumineuse à graines, de préférence aux autres, du fait de certaines de ses qualités :

— forte potentialité de rendement ;

- indéhiscence des gousses ;

- existence de plusieurs systèmes génétiques contrôlant la quasi-absence d'alcaloïde $(0,01 \%)$ (BERTHAUT \& SIXDENIER, 1977).

Mais cette espèce est particulièrement sensible à Pleiochaeta $s p$. et sa teneur en protéines ne dépasse pas $42 \%$.

L'hybridation de ces deux espèces devrait offrir des perspectives aux sélectionneurs, en permettant soit l'introgression de un ou plusieurs caractères intéressants, soit la création d'une espèce nouvelle.

Actuellement l'hybridation de ces deux espèces se heurte à une barrière d'incompatibilité prézygotique quand le parent mâle est $L$. albus et postzygotique si le parent mâle est $L$. mutabilis (WILliams et al., 1980). Et dans ce cas, l'arrêt de la croissance embryonnaire semble très précoce. Ces résultats ont été obtenus pour la seule variété Kievsky croisée par les génotypes $\mathrm{F} 82$ et $\mathrm{C} 82$, dans des conditions de serre. Nous avons donc décidé d'étudier l'importance des facteurs génétiques sur la "réussite » de ces croi- sements. Nous avons aussi pratiqué ces croisements soit au champ soit en serre. Par « réussite » nous entendons aussi bien le taux de nouaison après fécondation, que le rendement en graines contenant des embryons susceptibles d'être sauvés par culture in vitro. Pour le moment nous ne savons pas sauver des embryons, issus d'autofécondation de $L$. albus et L. mutabilis, de moins de 200 à $500 \mu \mathrm{m}$ (Vuillaume \& HOFF, 1986).

\section{MATÉRIEL ET MÉTHODE}

\section{A. Matériel}

\section{Lupinus mutabilis (parent mâle) :}

- mutants à faible teneur en alcaloïde : D50, E50, C66 et 16-1-6 ;

- matériel à faible teneur en alcalö̈de provenant de Lusignan : LMD19, LMD60-8, LMD63, LMD64 et LMD64-8, issus des croisements entre les génotypes de Lusignan et ceux de VON BAER ;

- matériel sélectionné par BERTHAUT à Dijon, dans la collection de Lusignan et dont nous avons observé l'aptitude à la culture in vitro: LM34 et LM32 ; $\mathrm{O} 82$;

- matériel précoce fourni par WILliams : E82 et

- populations de la collection de Lusignan : LM24, LM168, LM226 et LM916.

Lupinus albus (parent femelle) :

- Il s'agit des variétés inscrites au catalogue : Amiga, Lublanc, Lucky ; et des variétés en cours de sélection à Lusignan : Blanca, Lucrop, Lutop et Ultra.

\section{B. Méthodes}

\section{Réalisation des croisements}

Les croisements sont réalisés aussi bien sur la tige primaire que sur les rameaux secondaires, tertiaires ou quaternaires. Il suffit de s'assurer que la plante ne porte pas trop de gousses par ailleurs.

Bien que $L$. albus soit une espèce autogame, les fécondations croisées sont possibles, ce qui nécessite la protection des inflorescences choisies par un voile de tulle jusqu'à leur préparation pour croisements.

La préparation des inflorescences femelles consiste en l'élimination de toutes les fleurs trop vieilles ou trop jeunes; les boutons floraux sont alors ouverts et débarrassés de leurs étamines (vérifier impérativement la position des anthères en dessous du stigmate).

On dépose sur le style un fragment de carène contenant les anthères du génotype choisi comme parent mâle. Le prélèvement est effectué sur des fleurs arrivées à maturité. Les inflorescences femelles sont à nouveau couvertes d'un voile de tulle, pendant 48 à 72 heures.

\section{Observations histologiques des graines}

Les échantillons prélevés sont fixés dans du F.A.A. (Formalin Acétique Acid) puis, après déshydratation progressive à l'alcool, ils sont inclus dans la paraffine. 
Les coupes sont de 7 à $15 \mu \mathrm{m}$.

Après déparaffinage les préparations sont colorées à l'hématoxyline (coloration des parois et nucléoles) et après régression, contrecolorées à l'orange $\mathrm{G}$ (coloration des parois et du contenu cytoplasmique).

\section{RÉSULTATS}

Dans tous les cas nous n'avons obtenu ni gousse ni graine de taille comparable à celles obtenues après autofécondation (fig. 1).

\section{A. Travaux réalisés en serre}

La première série d'essais réalisée entre fin mai et début juin (tabl. 1) permet de distinguer grossièrement 2 classes de génotypes mâles :

- moins de $25 \%$ de nouaison: LMD60, D50, E50, LMD64-8, LMD63 et LMD19 ;

- de $25 \%$ à $50 \%$ de nouaison : LMD64, C66 et 16-1-6.

Toutefois la validité de ces classes et donc des effets mâles auxquels on pourrait les assimiler peut être mise en cause dans la mesure où il y avait plusieurs opérateurs, plusieurs dates de croisement et des effectifs de croisements très hétérogènes.

La deuxième série de croisements réalisée en juin, montre que des interactions entre génotypes mâles et femelles peuvent exister (tabl. 2). Dans cet essai, une seule manipulatrice a travaillé dans un délai de quelques jours.

Dans les deux séries d'essais, des gousses immatures ont été récoltées trois à quatre semaines après croisement, et les ovules (fécondés ?) prélevés afin de tenter un sauvetage d'embryons immatures in vitro, selon les techniques précédemment décrites (VuILlaumE \& HOFF, 1986). Ceci sans aucun succès.

On observe que, au mieux, les ovules mesurent de 2 à $2,5 \mathrm{~mm}$ lors de la mise en culture et que très vite ils se déshydratent, jaunissent et se réduisent à de petites masses jaunâtres de moins de $1 \mathrm{~mm}$.

Sur les gousses laissées sur les plantes, 4 à 6 semaines après croisement on observe aussi la déshydratation des ovules (fig. 1).

L'étude histologique de quelques-uns de ces ovules nous a permis de voir que ceux-ci ne contenaient pas d'embryons nettement différenciés et que la zone embryonnaire était nécrosée (fig. $2 \mathrm{~A}$ et $2 \mathrm{~B}$ ).

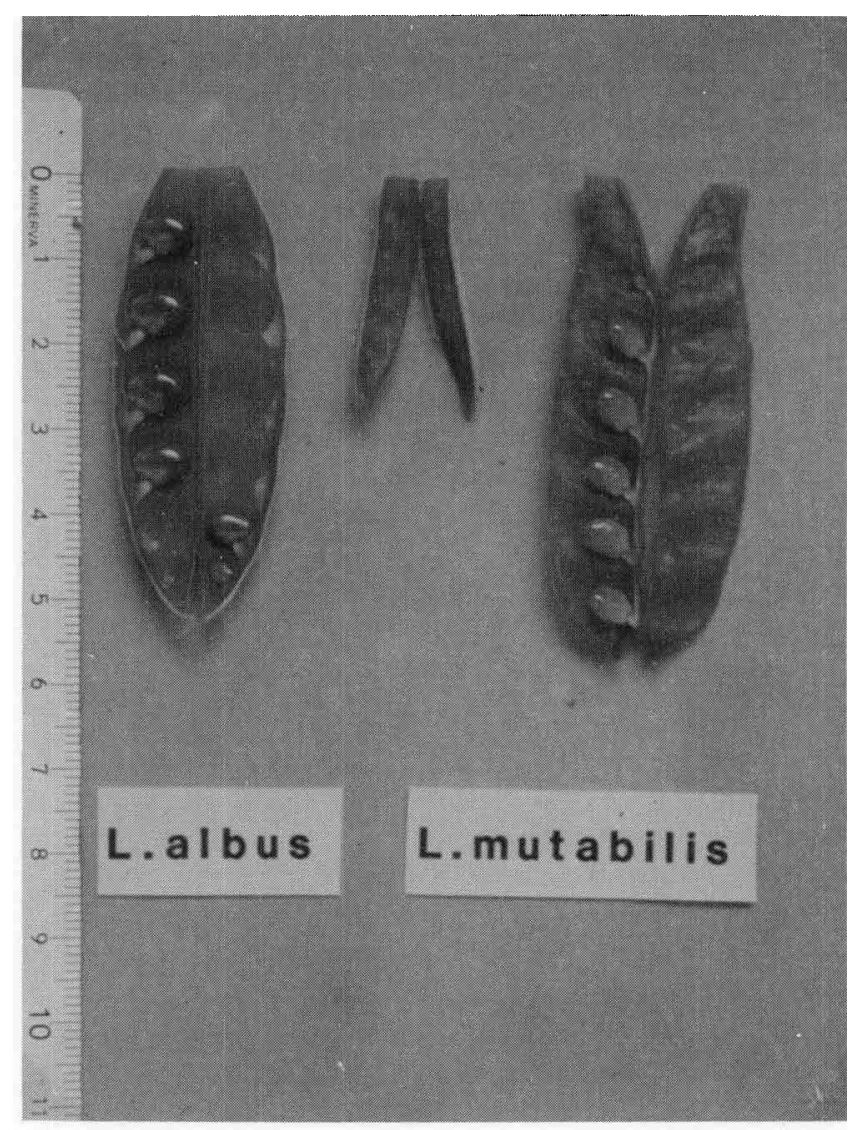

Figure 1

Gousses et ovules de Lupinus albus, L. mutabilis et, entre les deux, de leur hybride.

Les gousses issues de l'autofécondation des 2 espèces ont entre 3 et 4 semaines. La gousse provenant de l'hybridation interspécifique est âgée de 6 semaines, elle est netlement plus petite et contient 5 ovules (1 à gauche et 4 à droite) déshydratés et jaunissants.

Pods and ovules of Lupinus albus, L. mutabilis and of their interspecific hybrids.

For the two species, pods were sampled 3 to 4 weeks after selfpollination. For the interspecific hybrid, pod was sampled 6 weeks after pollination. This pod was smaller and contained 5 ovules dehydrated and yellowish (I on the left and 4 on the right).

\section{B. Travaux réalisés au champ}

La troisième série de croisements, réalisée au champ (tabl. 3) a été faite dans le but de mesurer des effets femelles et mâles sur le pourcentage de nouaison et le rendement en grains. La mesure des interactions est ici confondue avec l'erreur car il eût fallu réaliser au

TABLEAU 1

Première série d'essais, en serre, d'hybridation interspécifique entre Lupinus albus var. Lublanc $(Q)$ et différents génotypes de Lupinus mutabilis $\left({ }^{*}\right)$. Nombre de gousses 15 jours après la réalisation des croisements.

First attempt at interspecific hybridization, in the greenhouse, between Lupinus albus $c v$. Lublanc ( $\bigcirc)$ with different genotypes of Lupinus mutabilis $\left(O^{*}\right)$. Number of pods 15 days after pollination.

\begin{tabular}{|c|c|c|c|c|c|c|c|c|c|}
\hline Génotype & D50 & E50 & LMD & LMD & LMD & LMD & LMD & C66 & $16-1-6$ \\
\hline mâle & & & $64-8$ & $60-8$ & 63 & 64 & 19 & & \\
\hline $\mathrm{G} / \mathrm{F}$ & $3 / 18$ & $9 / 39$ & $11 / 53$ & $7 / 60$ & $15 / 78$ & $37 / 102$ & $16 / 83$ & $6 / 13$ & $17 / 49$ \\
\hline$\%$ & 16,7 & 23,1 & 20,7 & 11,7 & 19,2 & 36,3 & 19,2 & 46,2 & 34,7 \\
\hline
\end{tabular}


TABLEAU 2

Lupinus albus (१) $X$ Lupinus mutabilis $\left(O^{\circ}\right)$. Deuxième série d'essais réalisés en serre. Nombre de gousses 15 jours après la réalisation des croisements.

Lupinus albus ( $q$ ) $X$ Lupinus mutabilis $(o)$. Second greenhouse experiment. Number of pods 15 days after pollination.

\begin{tabular}{lcccc}
\hline $\begin{array}{l}\text { Génotype mâle } \\
\text { Génotype } \\
\text { Femelle }\end{array}$ & & LM34 & E82 & LM32 \\
LUBLANC & $\mathrm{G} / \mathrm{F}$ & $10 / 21$ & $5 / 8$ & $0 / 10$ \\
& $\%$ \% & 47,6 & 62,5 & 0 \\
AMIGA & $\mathrm{G} / \mathrm{F}$ & $11 / 42$ & $2 / 15$ & $2 / 22$ \\
& $\% \%$ & 26,2 & 13,3 & 9,0 \\
LUCKY & $\mathrm{G} / \mathrm{F}$ & - & $4 / 9$ & $0 / 22$ \\
& $\% \%$ & - & 44,4 & 0 \\
ULTRA & $\mathrm{G} / \mathrm{F}$ & $0 / 6$ & $0 / 5$ & $4 / 7$ \\
& $\%$ & 0 & 0 & 57,1 \\
\hline \hline
\end{tabular}

$\mathrm{G} / \mathrm{F}$ : nombre de gousses/nombre de fleurs croisées.

$\mathrm{G} / \mathrm{F}$ : number of pods/number of crossed flowers.

$\%$ : pourcentage de gousses formées par fleurs croisées.

$\%$ : pods per crossed flowers.

moins 3 séries de 20 croisements pour pouvoir l'estimer. Ceci était pratiquement irréalisable.

Le génotype femelle influe de manière significative sur le taux de nouaison 15 jours après le croisement, mais il n'a aucun effet sur la taille moyenne des gousses 1 mois après la date du croisement. Le génotype mâle n'a aucune influence sur ces 2 caractères comme le montrent les tableaux 4 et 5 d'analyse de variance.

Il est impossible de réaliser une analyse de variance sur le rendement en graines et sur la taille de celles-ci (tabl. 6). Cependant on peut conclure au vu de ces résultats, que le meilleur génotype femelle est Lucrop, suivi par Lutop, alors que le pourcentage de nouaison pour ces génotypes était faible au départ. Le meilleur génotype mâle est LM24 pour la taille moyenne des graines et 082 pour la quantité de graines dont la taille est supérieure à $2 \mathrm{~mm}$.

Toutes les graines obtenues ont été soumises à la préparation pour analyse histologique. Les coupes des graines dont la taille est supérieure à $4 \mathrm{~mm}$ sont souvent déchirées, car les téguments sont très durs et rendent difficile la fixation. Pour ces graines nous n'avons pas pu observer autre chose que des structures tégumentaires déchirées. Pour les graines plus petites la fixation est meilleure mais nous n'avons pu observer que des zones de nécrose à l'intérieur des téguments.

\section{CONCLUSION}

Il semble bien que des effets génétiques puissent moduler les résultats des hybridations interspécifiques entre $L$. albus et $L$. mutabilis. Cependant nous sommes toujours dans l'incapacité de savoir s'il y a formation d'embryons susceptibles d'être sauvés par culture in vitro.

Les essais en serre et au champ n'ont pas concerné les mêmes génotypes mâles et seul le génotype femelle Ultra est commun aux deux conditions de culture. Il est donc difficile de faire des comparaisons. L'obtention de graines contenant des embryons susceptibles

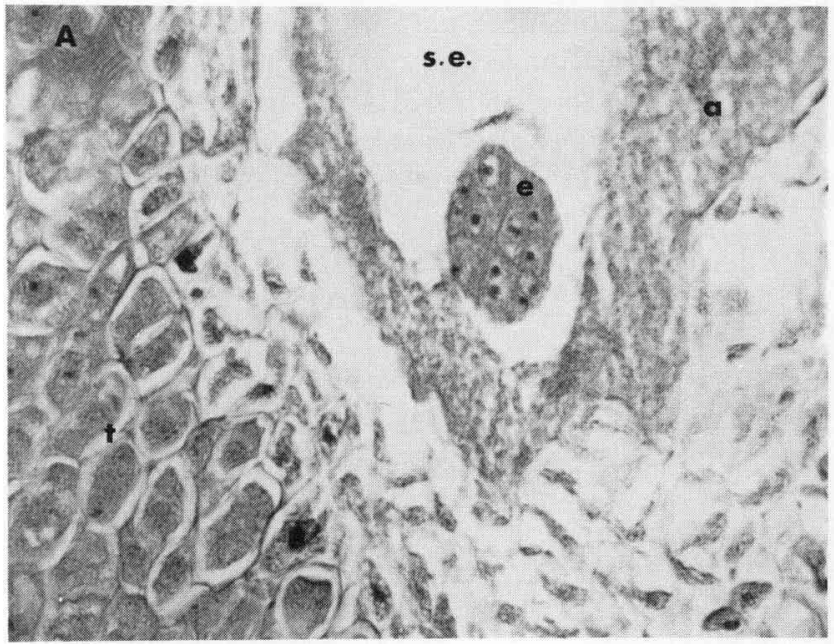

\section{a: albumen \\ e: embryon \\ t: tégument \\ s.e.: sac embryonnaire}

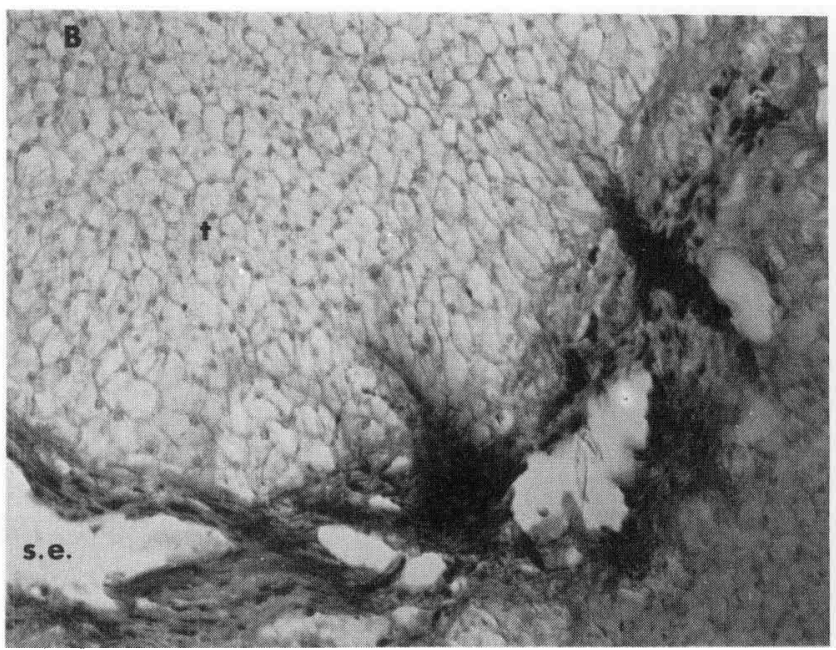

Figure $2 A$ el $B$

Coupes histologiques d'ovules de Lupinus albus et d'un hybride interspécifique de L. albus pollinisé par L. mutabilis.

$A$ : ovule de $\mathrm{L}$. albus mesurant $2,5 \mathrm{~mm}$, âgé d'environ 2 semaines et contenant un embryon au stade globulaire d'environ $45 \mu \mathrm{m}$.

$B$ : ovule d'un hybride interspécifique de $2 \mathrm{~mm}$, âgé de 18 jours et présentant dans la zone d'intertion de l'ovule un tissu nécrotique (zone à forte coloration).

Histological sections of Lupinus albus and of an interspecific hybrid of L. albus pollinated by L. mutabilis.

$A$ : L. albus ovule, $2.5 \mathrm{~mm}$ long, 2 weeks old, enclosing a globular embryo about $45 \mu \mathrm{m}$ long.

$B$ : interspecific ovule, $2 \mathrm{~mm}$ long, 18 days old, showing necrotic tissue in the embryonic insertion zone (highly stained zone).

d'être sauvés par culture in vitro n'a été possible qu'au champ et ce, quel que soit le génotype femelle.

Nous avons l'intention de reprendre les meilleurs génotypes et de procéder à des prélèvements systématiques de gousses dont les ovules seront soumis à une analyse histologique afin de savoir enfin s'il y a ou non formation d'embryons.

Un essai en chambre climatisée sera aussi réalisé afin de tenter de définir les conditions optimales de succès. 
TABLEAU 3

Lupinus albus $(\odot) X$ Lupinus mutabilis $\left({ }^{\circ}\right)$. Essai au champ réalisé le 1/7/1985. Lupinus albus (q) $X$ Lupinus mutabilis $(\sigma)$. Field experiment of $1 / 7 / 1985$.'

\begin{tabular}{|c|c|c|c|c|c|c|c|c|c|c|c|}
\hline \multicolumn{2}{|c|}{$\begin{array}{l}\text { Génotype mâle } \\
\text { Génotype femelle }\end{array}$} & \multicolumn{2}{|c|}{ LM 916} & \multicolumn{2}{|c|}{ LM 168} & \multicolumn{2}{|c|}{ LM 24} & \multicolumn{2}{|c|}{ LMO 82} & \multicolumn{2}{|c|}{ LM 226} \\
\hline & Date & $\mathrm{G}$ & $\mathrm{T}$ & $\mathrm{G}$ & $\mathrm{T}$ & G & $\mathrm{T}$ & $\mathrm{G}$ & $\mathrm{T}$ & $\mathrm{G}$ & $\mathrm{T}$ \\
\hline ULTRA & $\begin{array}{r}1 / 7 \\
10 / 7 \\
14 / 7 \\
23 / 7 \\
2 / 8 \\
21 / 8\end{array}$ & $\begin{array}{c}20 \\
15^{1} \\
13 \\
11^{3} \\
9 \\
7\end{array}$ & $\begin{array}{l}10,6 \\
14,5 \\
17,1 \\
18,6 \\
18,6\end{array}$ & $\begin{array}{c}18 \\
16 \\
14^{2} \\
10^{3} \\
4 \\
4\end{array}$ & $\begin{array}{c}10,6 \\
13,4 \\
14,0 \\
15,5 \\
-\end{array}$ & $\begin{array}{c}17 \\
14 \\
11^{2 *} \\
9^{3} \\
6 \\
6\end{array}$ & $\begin{array}{r}9,0 \\
12,7 \\
16,6 \\
19,0 \\
19,0\end{array}$ & $\begin{array}{r}15 \\
15 \\
13 \\
12 \\
11 \\
9\end{array}$ & $\begin{array}{l}10,7 \\
16,5 \\
17,7 \\
18,6 \\
18,5\end{array}$ & $\begin{array}{c}20 \\
14 \\
12 \\
9^{3} \\
9 \\
8\end{array}$ & $\begin{array}{l}10,2 \\
14,3 \\
18,8 \\
19,7 \\
20, C\end{array}$ \\
\hline BLANCA & $\begin{array}{r}1 / 7 \\
10 / 7 \\
14 / 7 \\
23 / 7 \\
2 / 8 \\
21 / 8\end{array}$ & $\begin{array}{l}21 \\
20 \\
17^{2 *} \\
14 \\
12 \\
11\end{array}$ & $\begin{array}{l}10,5 \\
14,3 \\
16,8 \\
18,7 \\
18,8\end{array}$ & $\begin{array}{l}20 \\
18 \\
17 \\
15 \\
10 \\
10\end{array}$ & $\begin{array}{l}10,4 \\
13,4 \\
15,7 \\
19,3 \\
19,3\end{array}$ & $\begin{array}{r}15 \\
11 \\
10 \\
10 \\
7 \\
7\end{array}$ & $\begin{array}{r}9,9 \\
13,2 \\
15,3 \\
20,1 \\
21,1\end{array}$ & $\begin{array}{l}20 \\
19 \\
18^{2} \\
16^{3} \\
14 \\
14\end{array}$ & $\begin{array}{l}11,2 \\
16,2 \\
17,0 \\
18,6 \\
18,3\end{array}$ & $\begin{array}{l}22 \\
21 \\
21 \\
17^{3} \\
17 \\
13\end{array}$ & $\begin{array}{l}12,2 \\
16,1 \\
19,5 \\
20,1 \\
20,4\end{array}$ \\
\hline LUTOP & $\begin{array}{r}1 / 7 \\
10 / 7 \\
14 / 7 \\
23 / 7 \\
2 / 8 \\
21 / 8\end{array}$ & $\begin{array}{r}15 \\
6 \\
6 \\
6 \\
6 \\
4\end{array}$ & $\begin{array}{r}8,3 \\
12,5 \\
16,2 \\
17,3 \\
16,8\end{array}$ & $\begin{array}{r}15 \\
9 \\
5 \\
5 \\
4 \\
3\end{array}$ & $\begin{array}{r}7,4 \\
12,6 \\
14,4 \\
18,8 \\
20,0\end{array}$ & $\begin{array}{r}15 \\
8 \\
8 \\
5 \\
5 \\
5\end{array}$ & $\begin{array}{r}7,1 \\
10,4 \\
11,8 \\
20,1 \\
21,0\end{array}$ & $\begin{array}{c}15 \\
12 \\
11^{2} \\
7^{3} \\
5 \\
5\end{array}$ & $\begin{array}{r}9,1 \\
12,5 \\
16,6 \\
19,6 \\
20,4\end{array}$ & $\begin{array}{c}16 \\
13 \\
11^{2} \\
9 \\
6 \\
4\end{array}$ & $\begin{array}{r}7,4 \\
8,7 \\
10,7 \\
13,2 \\
-\end{array}$ \\
\hline LUCROP & $\begin{array}{r}1 / 7 \\
10 / 7 \\
14 / 7 \\
23 / 7 \\
2 / 8 \\
21 / 8\end{array}$ & $\begin{array}{r}15 \\
10 \\
9 \\
8 \\
8 \\
8\end{array}$ & $\begin{array}{r}9,3 \\
13,8 \\
17,8 \\
18,9 \\
19,0\end{array}$ & $\begin{array}{c}15 \\
14 \\
10^{2 *} \\
8 \\
6 \\
6\end{array}$ & $\begin{array}{r}9,7 \\
12,6 \\
16,1 \\
19,9 \\
20,2\end{array}$ & $\begin{array}{c}15 \\
12 \\
11^{2} \\
9 \\
7 \\
7\end{array}$ & $\begin{array}{r}9,8 \\
13,2 \\
18,0 \\
22,7 \\
-\end{array}$ & $\begin{array}{r}17 \\
12 \\
9 \\
7 \\
6 \\
3\end{array}$ & $\begin{array}{r}9,3 \\
13,0 \\
20,3 \\
20,0 \\
20,7\end{array}$ & $\begin{array}{r}15 \\
11 \\
10 \\
8 \\
8 \\
7\end{array}$ & $\begin{array}{r}8,9 \\
12,9 \\
13,0 \\
15,4 \\
-\end{array}$ \\
\hline
\end{tabular}

Hybridations interspécifiques. Essai au champ réalisé le $1^{\text {er }}$ juillet 1985.

$\mathrm{G}$ : Nombre de gousses et $\mathrm{T}$ : taille moyenne des gousses en millimètres.

1: 2 gousses sont prélevées le $12 / 7$ pour observation histologique des ovules.

2 : 2 gousses sont prélevées le 16/7 pour culture in vitro (pour le croisement BLANCA X LM 916 une scule gousse a été prélevée).

3: 1 gousse est prélevée le $23 / 7$ pour électrophorèse des ovules.

* : autofécondations observées.

ULTRA X LM 24 : les 2 autofécondations sont repérées dès le 14/7, elles sont donc prises en considération pour la taille moyenne des gousses au $10 / 7$ uniquement.

BLANCA X LM 916: 2 autofécondations sont repérées au 14/7.

LUCROP X LM 916: 1 autofécondation est repérée au 14/7.

Interspecific hybridization. Field experiments of $1 / 7 / 85$.

$\mathrm{G}$ : number of pods and $\mathrm{T}$ : pod mean size in $\mathrm{mm}$.

1: 2 pods taken off for histological observation, on 12/7.

$2: 2$ pods taken off for in vitro culture on 16/7 (only one for the cross BLANCA X LM 916).

$3: 1$ pod taken off for electrophoresis on $23 / 7$

* : selfcrosses.

ULTRA X LM $24: 2$ selfcrosses observed on 14/7 and so included in the mean size of pods measured on $10 / 7$.

BLANCA X LM 916:2 selfcrosses observed on 14/7.

LUCROP X LM 916: 1 selfcross observed on 14/7.

TABLEAU 4

Analyse de la variance des pourcentages de nouaison 15 jours après croisement.

Analysis of variance of \% fruit set 15 days after pollination.

\begin{tabular}{lrrrr}
\hline \hline & $\begin{array}{c}\text { Somme } \\
\text { des carrés }\end{array}$ & $\begin{array}{c}\text { Degré } \\
\text { liberté }\end{array}$ & $\begin{array}{c}\text { Carré } \\
\text { moyen }\end{array}$ & \\
\hline Variation totale & 4778,92 & 19 & & \\
Effet femelle & 2418,54 & 3 & 806,18 & $4,93 *$ \\
Effet mâle & 398,50 & 4 & 99,62 & $<1,00 \mathrm{NS}$ \\
Résiduelle & 46,58 & 12 & 3,88 & \\
\hline
\end{tabular}

\section{TABLEAU 5}

Analyse de la variance de la taille moyenne des gousses I mois après croisement.

Analysis of variance of mean pod size, one month after pollination.

\begin{tabular}{|c|c|c|c|c|}
\hline & $\begin{array}{c}\text { Somme } \\
\text { des carrés }\end{array}$ & $\begin{array}{l}\text { Degré } \\
\text { liberté }\end{array}$ & $\begin{array}{l}\text { Carré } \\
\text { moyen }\end{array}$ & \\
\hline Variation totale & 80,69 & 19 & & \\
\hline Effet femelle & 9,42 & 3 & 3,14 & $<1,00 \mathrm{NS}$ \\
\hline Effet mâle & 24,69 & 4 & 1,59 & $<1,00 \mathrm{NS}$ \\
\hline Résiduelle & 46,58 & 12 & 3,88 & \\
\hline
\end{tabular}


TABLEAU 6

Nombre de graines $(G)$ et, taille des graines $(T)$ récoltées mesurée en millimètres.

Number of seeds harvested (G) and their size $(T)$ in $\mathrm{mm}$.

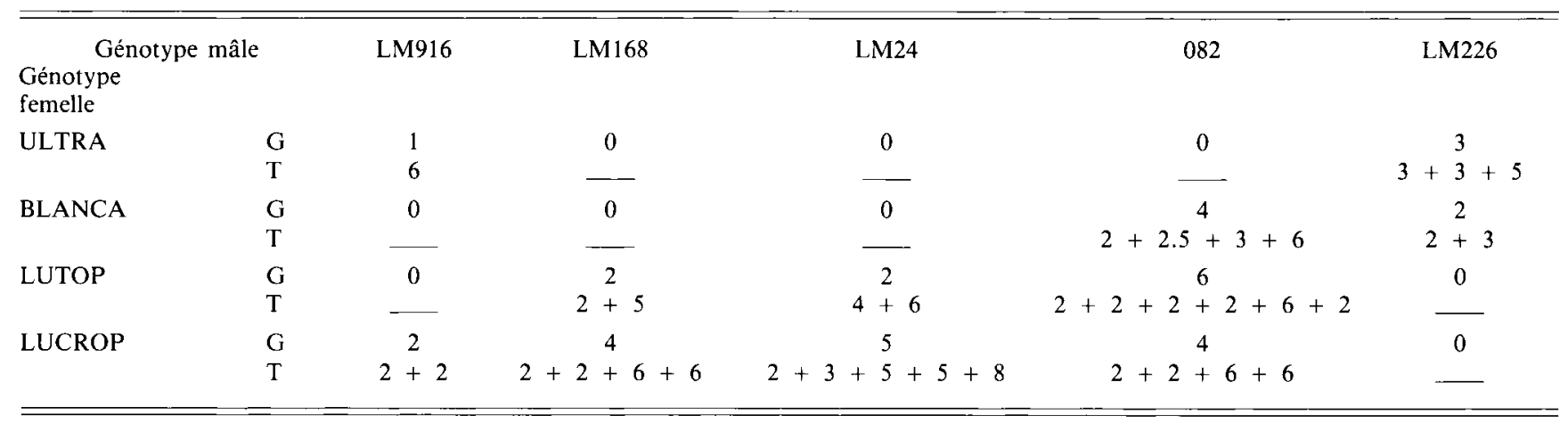

\section{REMERCIEMENTS}

Nous exprimons nos plus vifs remerciements à Madame ALABOUVETTE du Laboratoire de Botanique et d'Ecologie de l'Université de Dijon, sans laquelle le travail histologique n'aurait pas été réalisé.

\section{RÉFÉRENCES BIBLIOGRAPHIQUES}

Berthaut J., Sixdenier G., 1977. Les lupins - mise au point et premiers résultats sur les cultures de printemps. In : « Nouvelles des fourrages à l'I.N.R.A. ", 390 p., 343-346.

Blanco O., 1984. El germoplasma de Lupinus mulabilis en Sudamerica. In « $3^{e}$ Congrès International du Lupin », La Rochelle, 48 juin $1984,160-164$

Gladstone J. S., 1978. Recent development in the understanding, improvement and use of Lupinus. In : "Advances in Legume science ". Summerfield R. J. \& Bunting A. H. Eds., 603-611.

Lenoble M., Papineau J., 1977. Evaluation des différentes espèces de lupins à graine pour la production des protéines. In : «Nouvelles des fourrages de l'I.N.R.A. ", 390 p., 341-343.

Von Baer E., 1984. Plant breeding results of Lupinus albus and Lupinus mutabilis in southern Chile. In « $3^{e}$ Congrès International du Lupin », La Rochelle, 4-8 juin 1984, 545-547.
Von Baer E., Gross R., 1983. Sweet strains of Lupinus mutabilis. Zeitschrift für Pflanzenzücht., 91, 334-337.

Vuillaume E., Hoff T., 1986. Développement in vitro d'embryons immatures de Lupinus albus L. et Lupinus mutabilis L. par culture de gousses, d'ovules ou d'embryons isolés. Agronomie 6 (10), 925930.

Williams W., 1984. Lupins in crop production. In : "Outlook on agriculture ", Vol. 13 (2), 69-76.

Williams W., Akhtar M. A., Faluyi M., 1980. Cross compatibility between European and American Lupinus species. Bot. J. Linnean Soc., 81, 225-232.

Williams W., Harrison J. E. M., Jayasekera S., 1984. Genetical control of alkaloid production in Lupinus mutabilis and the effect of mutant allele mutal isolated following chemical mutagenesis. Euphytica, 33, 811-817. 